\title{
Transcriptomic convergence despite genomic divergence drive field cancerization in synchronous squamous tumors
}

Chin-Ann Johnny Ong ( $\nabla$ johnny.ong.c.a@singhealth.com.sg)

National Cancer Centre Singapore https://orcid.org/0000-0003-4573-7738

Nicholas B Shannon

National Cancer Centre Singapore

Weng Khong Lim

Duke-NUS Medical School

Qiu Xuan Tan

National Cancer Centre Singapore https://orcid.org/0000-0002-5700-1739

Daniel R.Y. Yap

National Cancer Centre Singapore

Sze Min Lek

National Cancer Centre Singapore

Joey W.S. Tan

National Cancer Centre Singapore

https://orcid.org/0000-0001-6620-6085

Shih Jia J. Tan

National Cancer Centre Singapore

Josephine Hendrikson

National Cancer Centre Singapore

Wai Har $\mathrm{Ng}$

National Cancer Centre Singapore

Ying Liu

National Cancer Centre Singapore

Gillian $\mathbf{N g}$

National Cancer Centre Singapore

Kelvin K.N. Koh

National Cancer Centre Singapore

Cedric C.Y. Ng

National Cancer Centre Singapore

Vikneswari Rajasegaran

National Cancer Centre Singapore

Choon Kiat Ong 
National Cancer Centre Singapore

\section{Tony K.H Lim}

Singapore General Hospital

\section{Bin Tean Teh}

National Cancer Centre Singapore

\section{Oi Lian Kon}

National Cancer Centre Singapore

Claramae S. Chia

National Cancer Centre Singapore https://orcid.org/0000-0001-5540-2646

\section{Khee Chee Soo}

National Cancer Centre Singapore

\section{N. Gopalakrishna lyer}

National Cancer Centre Singapore

\section{Case Report}

Keywords: field cancerization, field change, genomic, transcriptomic, aerodigestive tract tumors

Posted Date: March 9th, 2021

DOI: https://doi.org/10.21203/rs.3.rs-308860/v1

License: (c) (i) This work is licensed under a Creative Commons Attribution 4.0 International License. Read Full License 


\section{Abstract}

Field cancerization is suggested to arise from imbalanced differentiation in individual basal progenitor cells leading to clonal expansion of mutant cells that eventually replace the epithelium, although without evidence. Through deep sequencing analyses, we characterized the genomic and transcriptomic landscapes of field change in two patients with synchronous aerodigestive tract tumors. Our data support the emergence of numerous genetic alterations in cancer-associated genes but refutes the hypothesis that founder mutation(s) underpin this phenomenon. Instead, our analyses suggest a common etiologic factor defined by mutational signatures and/or transcriptomic convergence, which could provide a therapeutic opportunity.

\section{Background}

Field cancerization occurs when oral squamous cell carcinomas (SCCs) arise from a background of histologically normal but functionally abnormal tissues that develop a high incidence of multi-centric tumors. Herein, using valuable tumor and adjacent histologically normal tissue samples for integrative genomic and transcriptomic analysis, we provide insights into clinical field cancerization, novel therapeutic options, and comprehensive surveillance.

\section{Case reports}

Mr P (HN129) was a 38-year-old smoker (10 pack-years) diagnosed with three synchronous SCCs involving the left pyriform sinus, post cricoid space, and lower esophagus. Ms S (HN146) was a 50-yearold smoker (20 pack-years) diagnosed with two synchronous SCCs involving the right pyriform sinus and upper esophagus. Both patients underwent total pharyngo-laryngectomy (TPLE) with gastric pull-up. Histological assessment of both Mr P and Ms $\mathrm{S}^{\prime}$ tumors revealed histologically distinct tumors and no gross malignant change within the intervening mucosa. $\mathrm{Mr} \mathrm{C}$ (HN49) was a 60-year-old smoker (10 packyears) and alcoholic. He developed metachronous supraglottic SCC 3 years after subtotal glossectomy with neck dissection for SCC of the tongue.

\section{Results}

Genomic divergence is greater in synchronous than metachronous tumors

Genomic alterations were determined by whole exosome sequencing (WES) and array comparative genomic hybridization (aCGH) on bulk samples of paired tumor and adjacent normal mucosa from each patient (Supplementary Table S1). WES results demonstrated lower overall number of single nucleotide variants (SNVs) in adjacent normal mucosa compared to tumors in all three patients (Fig. 1a and 1b). The median (range) SNV counts of synchronous tumors (HN129 and HN146) were 197 (14\%) and 91 (23\%), respectively, while that of the metachronous tumor (HN49) was lower at 63 (22\%) (Fig. 1a). Our analysis of shared genetic alterations within matched tumor-normal combinations of patients with synchronous tumors showed no shared mutations among the different tumors of each patient (Fig. 1b), 
while only one shared mutation was identified in the metachronous tumors. Additionally, there were no shared copy number variants (CNVs) among synchronous tumors or the cognate matched intervening mucosa (Fig. 1c).

\section{Mutational signatures defining early events in field cancerization}

De novo mutational signature analysis ${ }^{1,2}$ revealed common trends among the three patients, such as the prevalence of Signatures 1 and 16 (Fig. 1d). We noted that Signature 4 (usually attributable to smoking) was prevalent in HN49 but not in the two synchronous patients. Contrastingly, Signature 3 dominated in both patients with synchronous tumors. In breast and pancreatic cancers, Signature 3 is strongly associated with germline or somatic BRCA1/BRCA2 mutations, ${ }^{3}$ although this was not observed in these two patients.

\section{Paradoxical genomic divergence with transcriptomic convergence in synchronous tumors}

We subjected matching sets of adjacent mucosa and tumor samples of all three patients to RNA sequencing. Hedgehog $(\mathrm{Hh})$ pathway was identified as the key signaling pathway in synchronous cancers (normalized enrichment score $(\mathrm{NES})=1.77, p$-value $=0.002$ ) but was not significantly upregulated in metachronous tumors (NES $=1.09, p$-value $=0.324$ ) (Fig. 1e). To validate the clinical relevance of $\mathrm{Hh}$ upregulation, we performed immunohistochemical (IHC) staining on a cohort of head and neck SCC (HNSCC) patients ( $n=328$ ) (Supplementary Fig. S1b). Approximately $25 \%$ of patients demonstrated upregulated $\mathrm{Hh}$ signaling, which was associated with poor prognosis $(p=0.014)$ (Supplementary Fig. S1c). This was similar in an independent cohort of HNSCC patients in The Cancer Genome Atlas (TCGA) database $^{4}(p=0.015)$ (Supplementary Fig. S1d).

Activation of Hedgehog signaling in basal cells as a putative transcriptomic driver of synchronous tumors

To determine the distribution of subsequent genetic events, we performed laser capture microdissection (LCM) of normal and dysplastic mucosa, as well as of adjacent synchronous tumors in HN129. DNA was extracted from tumor, basal, and squamous compartments, and subjected to targeted sequencing using a panel of mutations detected by WES in HN129 and other known oncogenic drivers of HNSCC (Supplementary Table S1 and Fig. 1f). In 'normal' mucosa immediately adjacent to tumors, we found specific TP53 mutations shared between the basal and tumor areas, or basal and squamous layers (Fig. 1f). In other situations, TP53 mutations were discordant between tumor and adjacent basal layer. Variant allele frequencies were significantly higher in tumors compared to histologically normal or dysplastic regions. Furthermore, the basal layer had fewer SNVs (apart from TP53) compared to squamous layers or tumors. This suggests that TP53 mutations are early events in the basal layer, which gain subsequent alterations that drive it either to terminal differentiation in the squamous layer or tumor formation (Supplementary Fig. S1e). Contrastingly, certain late genetic events are only seen in tumors and never in the basal or squamous layers (e.g. NFE2L2) (Fig. 1a and 1f). 


\section{Discussion}

Our data support the emergence of numerous genetic alterations in cancer-associated genes, likely from the basal layer, but refutes the hypothesis that either a single or a limited panel of founder mutations, where tumors growing in a cancerized field originate from a single malignant cell, as an early event in synchronous field cancerization. ${ }^{5}$ Our analyses suggest a common etiologic factor defined by mutational signatures and/or transcriptomic convergence. Targeted resequencing of subcellular components demonstrated distinct mutational profiles of squamous epithelium and tumors coupled with a paucity of mutations in the basal cell layer, suggesting that the latter likely contributes the cell of origin for synchronous tumors.

Our transcriptomic data support the notion that the concept of field cancerization relates more to etiologies that alter the microenvironment and drive mutagenic events during carcinogenesis, rather than actual initiating founder mutations. ${ }^{6,7}$ Transcriptomic changes could pre-date genomic alterations, reflecting cellular phenotypic responses to the initial mutagenic insult. This 'transcriptomic convergence' may provide a clue to the 'cell of origin' that drives field cancerization, and unravel therapeutic opportunities that prevail across all tumors in a field, regardless of genetic diversity. Alternatively, routine screening procedures such as sampling of the esophagus via the Cytosponge could help to document genomic divergence and incorporate testing for Shh signaling pathway activation, thereby capturing clinically relevant oncogenic factors beyond those imputed by classical theory. ${ }^{8}$

\section{Materials And Methods}

Surgical samples from these three patients enabled integrative analysis of synchronous tumors and matched adjacent normal mucosa. This study was approved by the SingHealth Centralized Institutional Review Boards (CIRB 2008/467/B, 2011/678/B, and 2007/438/B). Written informed consent was obtained from all patients in accordance to the study protocols. Detailed methodology is described in the supplementary data.

\section{Declarations}

\section{Data Availability}

The datasets generated during and/or analysed during the current study are available from the corresponding author on reasonable request.

\section{Acknowledgements}

This initiative is funded by SingHealth Duke-NUS Academic Medical Centre, facilitated by Joint Officer of Academic Medicine (JOAM). It is an initiative of Surgery Academic Clinical Program (SACP) under SACP Seed grant and the Surgeon-Scientist Strategic Start-Up Grant. Furthermore, this work is also supported by NCCS Research Fund and SingHealth Oncology Academic Clinical Program under the Cancer 
Collaborative Scheme. NGI is supported by National Medical Research Council Clinician-Scientist Awards (NMRC/CSA-INV/0011/2016 and MOH-000325-00). CAJO is supported by the National Medical Research Council Transition Award (NMRC/TA/0061/2017). All the funding sources had no role in the study design, data interpretation or writing of the manuscript.

\section{Authors' contributions}

Conceptualization: CAJO and NGI; Methodology: CAJO and NGl; Validation: NBS, WKL, QXT, DRYY, SML, JWST, SJJT, JH, WHN, YL, GN, KKNK, CCYN, VR, CKO, TKHL; Formal analysis: NBS, WKL, QXT, DRYY, SML, JWST, SJJT, JH, WHN, YL, GN, KKNK, CCYN, VR, CKO, TKHL; Investigation: NBS, WKL, QXT, DRYY, SML, JWST, SJJT, JH, WHN, YL, GN, KKNK, CCYN, VR, CKO, TKHL; Resources: CAJO and NGI; Data curation: QXT, DRYY, SML, SJJT; Writing - original draft: CAJO, QXT, DRYY, BTT, OLK, CSC, KCS and NGI; Writing review \& editing: CAJO, NBS, WKL,QXT, DRYY, SML, JWST, SJJT, JH, WHN, YL, GN, KKNK, CCYN, VR, CKO, TKHL, BTT, CSC, KCS and NGI; Visualization: CAJO, QXT and NGI; Supervision: CAJO, BTT, OLK, CSC, KCS and NGl; Project administration: CAJO and NGl; Funding acquisition: CAJO and NGI.

\section{Competing interests}

The authors declare that they have no competing interests.

\section{References}

1. Alexandrov, L. B., Nik-Zainal, S., Wedge, D. C., Campbell, P. J. \& Stratton, M. R. Deciphering signatures of mutational processes operative in human cancer. Cell Rep3, 246-259 (2013).

2. Nik-Zainal, S. et al. Mutational processes molding the genomes of 21 breast cancers. Cel/149, 979993 (2012).

3. Alexandrov, L. B. et al. Signatures of mutational processes in human cancer. Nature500, 415-421 (2013).

4. Lawrence, M. S. et al. Comprehensive genomic characterization of head and neck squamous cell carcinomas. Nature517, 576-582 (2015).

5. Curtius, K., Wright, N. A. \& Graham, T. A. An evolutionary perspective on field cancerization. Nat. Rev. Cancer18, 19-32 (2018).

6. Lochhead, P. et al. Etiologic field effect: reappraisal of the field effect concept in cancer predisposition and progression. Mod. Pathol.28, 14-29 (2015).

7. Frede, J., Greulich, P., Nagy, T., Simons, B. D. \& Jones, P. H. A single dividing cell population with imbalanced fate drives oesophageal tumour growth. Nat. Cell Biol.18, 967-978 (2016).

8. Fitzgerald, R. C. Combining simple patient-oriented tests with state-of-the-art molecular diagnostics for early diagnosis of cancer. United European Gastroenterol J3, 226-229 (2015).

\section{Figures}




\section{a}

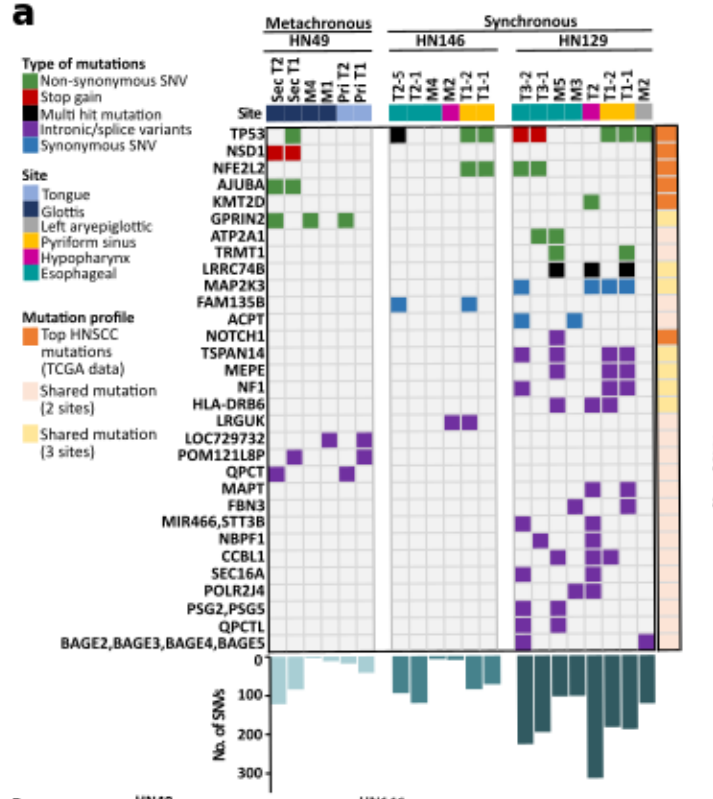

b
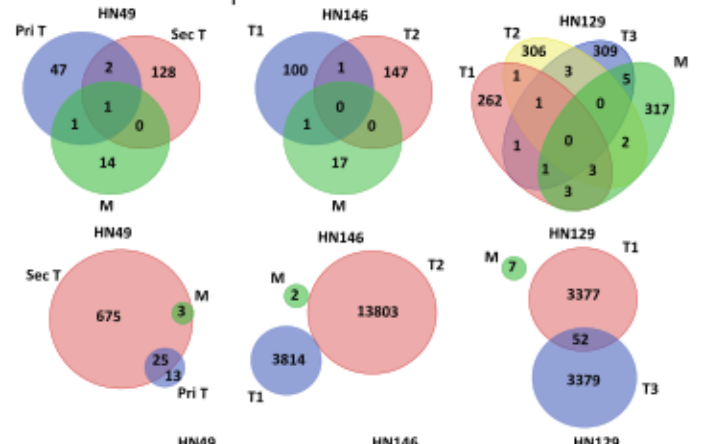

\section{d}
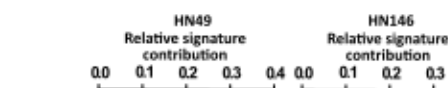
Signature 1.000 .0 .2 Signature 3 Signature 4 Signature 5 . $\frac{3}{2}$ Signature 8 ç Signature 8 Signature 17¿ Signature 19Unknown $\square$

e
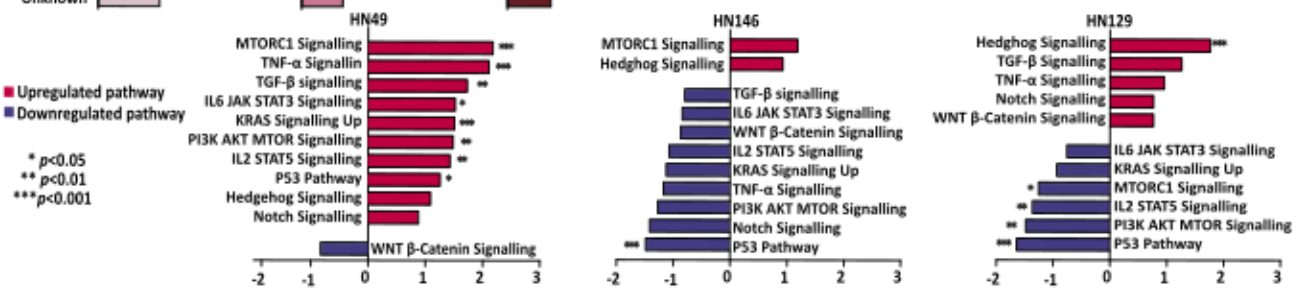

Figure 1

Synchronous tumors of the aerodigestive tract possess distinct tumor biology from that of metachronous tumors. a) Heatmap illustrating the mutational landscape of the samples derived from three distinct patients. Frequency of single nucleotide variants in tumor and adjacent normal mucosa indicate lower mutation counts in normal mucosa (M) compared to that of tumor (T) in all three patients. b) No shared mutations were identified between the normal mucosa and synchronous tumors while merely one shared 
mutation was identified in metachronous tumors and mucosa. c) Few copy number alterations are shared across the samples in both synchronous and metachronous tumors. d) Signature 3 was common to synchronous tumors whereas Signature 4 was unique to metachronous tumors. e) Differential modulation of signaling pathways is seen between synchronous (HN129) and metachronous (HN49) tumors via gene set enrichment analysis (GSEA). Normalized expression values are reflected in the bar charts, where $p$-value $<0.05$ is represented with blue (down-regulated) or red (up-regulated). Our data revealed that hedgehog pathway was significantly enriched in synchronous tumors. f) Synchronous tumors of the aerodigestive tract display a unique profile of genomic divergence and transcriptomic convergence. SNV, single nucleotide variants; $M$, mucosa; T, tumor; Shh, sonic hedgehog

\section{Supplementary Files}

This is a list of supplementary files associated with this preprint. Click to download.

- Supplementarynpjinal.docx 